\title{
Psyche trifft Körper - der komorbide Patient als echte Herausforderung der Interdisziplinarität
}

\author{
Mind Meets Body - The Comorbid Patient as a True Challenge \\ of Interdiscipinarity
}

Autor

Institut
Bernd Eikelmann

Klinik für Psychiatrie und Psychotherapeutische Medizin Karlsruhe

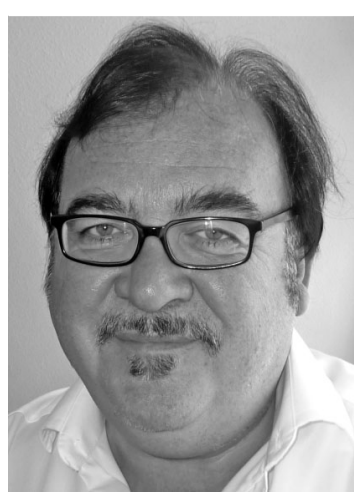

Prof. Dr. med. Bernd Eikelmann

\section{Bibliografie}

Dol http://dx.doi.org/ 10.1055/s-0030-1248573 Psychiat Prax 2010; 37: 265-267

(c) Georg Thieme Verlag KG Stuttgart · New York . ISSN 0303-4259

Korrespondenzadresse

Prof. Dr. med.

Bernd Eikelmann

Klinik für Psychiatrie und

Psychotherapeutische Medizin

Kaiserallee 10

76133 Karlsruhe

Bernd.Eikelmann@klinikum-

karlsruhe.de
Notruf des Konsilarztes an den Leiter einer psychiatrischen Fachabteilung an einem Krankenhaus der Maximalversorgung: Konsilpatienten soweit das Auge reicht, die Inanspruchnahme steigt kontinuierlich an - ich kann nicht mehr. In den zurückliegenden 25 Jahren hat sich etwas getan: psychiatrische Konsile waren früher (in meinem Fall in einer Universitätsklinik) vielfach vom Patienten unerwünschte, teils nur hingenommene oder sogar vor ihm getarnte Besuche am somatischen Krankenbett („Da kommt gleich ein junger Kollege aus der Nachbarklinik“), die häufig einen rein rechtlichen Hintergrund hatten oder der Abwehr von unmittelbaren Gefahren dienten. Heute ist das psychiatrische Konsil meist unproblematisch, sehr oft erwünscht und wird sogar zuweilen eisern nachgefragt. Wir treffen dabei Menschen aus der Mitte des Lebens und der Mitte der somatischen Medizin, will sagen, mit den gängigen Problemstellungen und Krankheitsbildern. Die 69-jährige Patientin mit dem HighRisk-Melanom, das vor einem halben Jahr operiert wurde, stellt sich vor und berichtet, dass sie vor 3 Jahren ihre Mutter verloren hat, auf die sie ihr ganzes privates Leben ausgerichtet hatte. Es folgt der 25-jährige junge Mann, der unter einer seltenen anatomischen Variation im Urogenitaltrakt leidet und durch Scham und Angst die letzten 3-4 Jahre überwiegend zu Hause vor seinem Computer verbracht hat. Der 50-jährige Mann, der eine Lippen-Kiefer-Gaumen-Spalte hat und ausführlich berichtet, wie sein erstes Lebensjahrzehnt von der Angst, nicht gemocht und aus jeder sozialen Situation ausgeschlossen zu werden, überschattet war. Die Reihe kann man fortsetzen. Ach ja, da war doch der 70-jährige Patient, der einen Defibrillator (ICD) eingepflanzt bekommen hat und mehrfach die Aktion des Gerätes erleben konnte: im Liegen auf dem Sofa hatte er die hohe Herzfrequenz wahrgenommen und den „Schock“ förmlich erwartet.
Insbesondere in Richtung älterer Patienten in der Inneren Medizin, der Onkologie und Kardiologie ergeben sich aktuell erhebliche Überschneidungsbereiche. Grob geschätzt dürften ein Drittel der kardiologischen Patienten und ca. 80\% der onkologischen Patienten psychische Auffälligkeiten bieten. Typische Patienten sind aber auch jene mit Tinnitus, mit „Fatigue“-Syndrom (z.B. im Rahmen eines Mammakarzinoms oder bei MS), mit Schlafstörungen, mit Schmerzen, der „neurologische“ Demenzkranke, der ParkinsonKranke, der polytraumatisierte Patient usw. Etwas allgemeiner ausgedrückt entstehen die Probleme unserer Patienten nicht entlang der Weiterbildungsordnung für Ärzte. Gehirn und Körper sind auch in Krankheiten vielmehr Einheit, als es die Organisation des Medizinbetriebes heute erwarten lässt. Psychische und körperliche Morbidität sind eng miteinander verbunden. In der Lübecker Allgemeinen Krankenhausstudie [1] wurden bei 400 internistischen und chirurgischen Patienten folgende punktuelle Häufigkeiten psychischer Störungen diagnostiziert: 46,5\% der internistischen Patienten erhielten eine psychiatrische Diagnose nach ICD-10, bei $16 \%$ wurde eine organische psychische Störung, bei $8 \%$ eine Störung durch psychotrope Substanzen, in 1\% eine Schizophrenie, in 9,5\% affektive Störungen und in $10,5 \%$ neurotische, Belastungs- und somatoforme Störungen angenommen. Eine aktuelle Übersicht zu Depression und Angst bei Bandscheibenoperierten ergab Raten von 21,5-49,3\% [2].

Aber auch die umgekehrte Denkrichtung bringt interessante Resultate. In ihrem Bericht „equal treatment: closing the gap“, der im Jahr 2006 publiziert wurde, fand eine Kommission, die das staatliche englische Gesundheitssystem auf Benachteiligungen untersuchte, dass Menschen mit Schizophrenie oder bipolarer Störungen im Vergleich mit der Allgemeinbevölkerung sehr viel 
häufiger einen Diabetes mellitus, eine koronare Herzkrankheit, Bluthochdruck oder einen Schlaganfall erleiden [3]. Im Übrigen tritt das in einem jüngeren Lebensalter auf und führt schneller zum Tode. Ähnliches gelte für Menschen mit Lernbehinderungen. Als Gründe wurden v.a. Armut, eine ungesunde Lebensweise, ein schlechter Zugang zum Gesundheitssystem und unerwünschte Wirkungen der psychiatrischen Medikation genannt [4,5]. Gerade für Patienten mit Schizophrenie konnte auch für Deutschland gezeigt werden, dass eine ungesunde Lebensweise eine Rolle spielt $[6,7]$. Karzinomerkrankungen wurden ebenfalls häufiger diagnostiziert, wobei besonders auf den Zusammenhang mit Nikotinabusus hingewiesen wurde. Auch in Deutschland haben Menschen mit schweren psychischen Erkrankungen eine verkürzte Lebenserwartung, während unser Gesundheitssystem vermutlich bessere Chancen bietet. Allerdings liegen hierzu Vergleichszahlen nicht vor. Gewiss ist aber, dass psychiatrische Krankenhauspatienten auch hier sozial exkludiert und somit umfassend benachteiligt sind [8].

Psychische Komorbidität bei körperlichen Erkrankungen und somatische Komorbidität bei psychischen Erkrankungen sind häufige Phänomene. $\mathrm{Zu}$ Beginn eines akuten Infektes tritt nicht selten ein psychisches Korrelat („Protopathisches Krankheitsgefühl“) mit Abgeschlagenheit, leichter depressiver Verstimmung und Antriebsstörung auf. Erschöpfung, Angst, Scham, Trauer, Schlafstörungen und kognitive Probleme kennzeichnen die Psychopathologie von onkologischen Patienten [9]. Depressive weisen ein erhöhtes Risiko für das Koronarsyndrom oder den Herzinfarkt auf [10]. Depressionen haben negativen Einfluss auf den Verlauf bereits bestehender kardiovaskulärer Erkrankungen. Schizophrenien, Depressionen und Angsterkrankungen erhöhen die kardiovaskuläre Sterblichkeit. Alles deutet darauf hin, dass eine zentrale Dysregulation bei diesen psychischen Störungen bestehen könnte und körperliche Komplikationen wahrscheinlicher macht. Umgekehrt erkranken viele Patienten mit einer ernsthaften körperlichen Gesundheitsstörung auf unterschiedlichen Wegen psychisch: ein Beispiel dafür sind Parkinson-Kranke, deren hohe Inzidenz für depressive Verstimmungen keineswegs nur aus den gestörten neurobiologischen Abläufen und dem Dopaminmangel abzuleiten ist.

\section{Der Dschungel der Versorgungspraxis - wer findet sich zurecht? \\ $\nabla$}

Wenn das alles so offenliegt und scheinbar sinnfällig ist, wieso kommen die entsprechenden Wahrnehmungen und Handlungsprogramme so schwer in Gang? George Engel könnte Genugtuung empfinden: somatopsychische Interferenzen gewinnen an wissenschaftlicher Evidenz, allein das „Soziale“ kommt in den Forschungen zu kurz. Die praktische Seite jedoch, heute weit überwiegend Konsiliar- und Liaisonpsychiatrie (und nicht -psychosomatik), steht nicht gerade im Mittelpunkt der wissenschaftlichen Diskussion oder der Versorgungsforschung [11-13]. Es gibt einige Protagonisten, die diese wichtige Brücke zwischen Psychiatrie und Somatik schlagen, die auf der Ebene der Fachgesellschaft unentwegt promoten und dennoch langsam resignieren. Sie haben es durchwegs schwer, in praxi das nötige Gehör zu finden. Warum ist das so? Die oben dargestellte Sichtweise, der ganze Mensch ist krank, es kommt zur Störung vieler somatopsychischer Regelkreise, ist integrativ und nicht spezialistisch, also zeitaufwendige Übersetzungsarbeit. Viele medizinische Teildisziplinen sind zwar interdisziplinär und kollegial vernetzt, je- doch ist die Verbindung zur psychosozialen Medizin schwerer zu halten; sie ist nämlich oft extraterritorial. Man könnte daher etwas pointiert formulieren: Konsile ja und sofort, Liaisondienste und interdisziplinäre Zusammenarbeit aber bitte später und nur, wenn es sein muss. Hier dürften die alten Gewohnheiten einerseits und das Stigma des Faches andererseits eine Rolle spielen [14].

Da hilft es auch nicht, dass viele positive Aspekte der Konsiliarund Liaisonarbeit wie Verbesserung der therapeutischen Ergebnisse, Verkürzung der Verweildauern und Kostenreduktion überzeugend nachgewiesen wurden [15]. Sodann: Konsildienste gehören zur Psychiatrie und verursachen zunächst Kosten dort, Liaisondienste werden häufiger der somatischen Disziplin zugeschlagen, unter deren Dach sie ausgeübt werden. Die Psychoonkologie ist z.B. Bestandteil des Brustzentrums, des CancerCenters usw. Die Mitarbeiter figurieren dort und haben dort ihre Erfolge. Viele Mitarbeiter betrachten Konsildienste nicht gerade als ihre Herzensangelegenheit. Sind sie jedoch hauptamtlich in einem psychoonkologischen Bereich tätig, dann können sie ihr Selbstbewusstsein und ihre Karriere zwar dort entwickeln, bezogen auf die Herkunftsklinik sind sie außen vor und vom Hauptkarrierepfad entfernt.

Die finanziellen Anreizsysteme für die interdisziplinäre Zusammenarbeit sind schwach. Durch die DRGs ist kein Anreiz entstanden, weil die Preise durch Konsile nicht wesentlich gesteigert werden können. Die seit Kurzem gültigen OPS-Codes für unser Fach sind zurzeit noch fiktional, beinhalten schöne neue Begrifflichkeiten („Somato-psychosomatisches ärztliches Gespräch“), während ihre konkreten finanziellen Auswirkungen unklar bleiben. Viel stärker haben sich z. B. in der Onkologie fachliche Gesichtspunkte und Zertifizierungen niedergeschlagen. Aus der Sicht einer psychiatrischen Klinik ist der Konsildienst daher ein fragwürdiges Zusatzgeschäft, die Liaison findet nicht bzw. auf der anderen Seite des Jordans statt und gerät doch allzu leicht aus dem Blickwinkel. Den Beteiligten muss es wie Cato mit seinem berühmten „ceterum censeo“ gehen; es gibt gute Konzepte, sie werden durch die wissenschaftliche Empirie vielfach bestätigt, die Praxis bleibt jedoch zersplittert. Kann sich der Medizinbetrieb eine solche Lücke leisten?

Ein weiteres Problem ist das komplizierte Verhältnis zwischen Psychiatrie/Psychotherapie und Psychosomatik. Hier ist nicht der Ort, die geschichtliche Entwicklung und den momentanen Stand zu analysieren, jedoch muss deutlich gesagt werden, dass ein fruchtbarer Dialog zwischen den Disziplinen bis in die Gegenwart kaum möglich ist. Vielmehr werden parallele und teils redundante Versorgungsstrukturen weiterhin entwickelt: Etwa 53000 akute psychiatrische Betten stehen 3000 akuten und 15000 psychosomatischen Reha-Betten gegenüber [16]. Die Kollegen können sich daher im Einzelfall überlegen, wohin sie ihren Patienten stationär einweisen, jedenfalls gilt das für die ICDDiagnosegruppen von F3-F6. Hier kann man zwischen gemeindenaher psychiatrischer Klinik, zuweilen gemeindenaher psychotherapeutischer Akutklinik, zwischen psychosomatischer Akutstation und im Grünen gelegener psychosomatischer Rehabilitationseinrichtung wählen. Nicht zu vergessen jene Tageskliniken, die sich der psychotherapeutischen Medizin oder Psychosomatik verschrieben haben [17]. Es ist nicht zuletzt die Konkurrenz um einzelne Patientengruppen, sondern die unterschiedliche Interpretation der Versorgungspflichten und diese organisatorische Redundanz, die die Integration der Psychiatrie/Psychotherapie in die somatische Medizin aufhalten. 
Eine alte Forderung aus dem Jahre 1975 kommt in Erinnerung. Die ersten 35 Jahre Gleichstellung von psychisch und somatisch Kranken und Integration der Psychiatrie in die somatische Medizin haben vielversprechend begonnen. Sie lassen aber auch erkennen, dass es sich um eine epochale Aufgabe handelt. George L. Engels biopsychosoziales Modell war eben nur der theoretische Vorgriff auf eine interdisziplinäre psychosomatische Praxis, die durch Menschen gestaltet und gelebt werden muss, deren innerer Kompass der Eigenständigkeit ihres Faches und dem Spezialistentum den Vorzug gibt [18]. Vielleicht ist es klüger mit der Blickrichtung psychische Komorbidität bei somatisch Kranken anzufangen und vielleicht ist die Honigfalle schneller errichtet, wenn man bereit ist, sich auf Begrifflichkeiten wie Burn-out, Fatigue- oder Fibromyalgiesyndrom einzulassen, bei denen es sich um phänomenologisch sichere, aber wissenschaftlich dubiose Entitäten zwischen Neurasthenie, Depression und somatoformer Störung handelt. Sie spielen in der Onkologie oder der Rheumatologie eine wichtige Rolle. Nicht zu vergessen sind aber die Protagonisten der Praxis; sie wollen Beachtung und Anerkennung. Konsiliarische Psychiater sind Ärzte mit hoher Fachkompetenz an einer wichtigen Nahtstelle. Sie stehen weder fachlich noch persönlich den Kollegen in der Somatik nach. Ihre Aufgabe braucht viel Motivation und immer noch Pioniergeist. Eins ist jedoch sicher: Ohne diesen Beitrag des Faches Psychiatrie/Psychotherapie ist moderne Medizin, gerade auch in der „High-tech“Variante, unmöglich oder sinnlos.

\section{Literatur}

1 Arolt V. Psychische Störungen bei Krankenhauspatienten - Eine epidemiologische Untersuchung zu Diagnostik, Prävalenz und Behandlungsbedarf psychiatrischer Morbidität bei internistischen und chirurgischen Patienten. Berlin: Springer, 1997

2 Zieger M, Schwarz R, König HH et al. Depression and anxiety in patients undergoing herniated disc surgery: relevant but underresearched - a systematic review. Cen Eur Neurosurg 2010; 71: 26-34
3 Disability Rights Commission. Equal treatment: closing the gap. NHS Evidence, Oktober 2006

4 Weinmann S, Read J, Aderhold V. Influence of antipsychotics on mortality in schizophrenia: systematic review. Schizophr Res 2009; 113: 111

5 Leucht S, Burkard T, Henderson J et al. Physical illness and schizophrenia: a review of the literature. Acta Psychiatr Scand 2007; 116 : 317333

6 Roick C, Schindler J, Angermeyer MC et al. Health habits of patients with schizophrenia: a general pattern? Neuropsychiatr 2008; 22: 100-111

7 Roick C, Fritz-Wieacker A, Matschinger $\mathrm{H}$ et al. Health habits of patients with schizophrenia. Soc Psychiatry Psychiatr Epidemiol 2007; 42: 268-276

8 Harter C, Nattermann L, Weber $H$ et al. Soziale Gleichstellung psychiatrischer und somatischer Krankenhauspatienten? Ein empirischer Vergleich. Nervenarzt 2008; 79 (Suppl. 4): 500

9 Cramer SM, Eikelmann B, Sautter Bihl ML. Psychische Komorbiditäten und Syndrome bei radioonkologischen Patienten - gibt es Unterschiede bei den einzelnen Tumortypen? Abstract A-253-0015-00499. 18. Jahreskonferenz der Deutschen Gesellschaft für Radioonkologie, 2010

10 Scherrer JF, Chrusciel T, Zeringue A et al. Anxiety disorders increase risk for incident myocardial infarction in depressed and nondepressed Veterans Administration patients. Am Heart J 2010; 159: 772-779

11 Borbé R, Flammer E, Borbé S et al. Sozialpsychiatrische Forschung - Entwicklung über die letzten 10 Jahre im Spiegel deutschsprachiger Zeitschriften. Psychiat Prax 2009; 36: 362-367

12 Riedel-Heller SG, Bramesfeld A, Roick C et al. Der Ruf nach mehr Versorgungsforschung. Psychiat Prax 2008; 35: 157-159

13 Bramesfeld A, Riedel-Heller SG. Prioritäre Themen in der Versorgungsforschung zur psychischen Gesundheit. Psychiat Prax 2008; 35: 315317

14 Schomerus G. Steine auf dem Weg - Stigma und Hilfesuchverhalten. Psychiat Prax 2009; 36: 53-54

15 Diefenbacher A, Grubel H. Aktuelle Konsiliarpsychiatrie und -psychotherapie. Stuttgart: Thieme, 1999

16 Salize HJ, Rössler W, Becker T. Mental health care in Germany. Current state and trends. Eur Arch Psychiatry Clin Neurosci 2007; 257: 92-103

17 Eikelmann B. Tagesklinische Behandlung in der Psychiatrie. Nervenarzt 2010; 81: 355-363

18 Engel GL. From biomedical to biopsychosocial: being scientific in the human domain. Psychosomatics 1997; 38: 521-528 